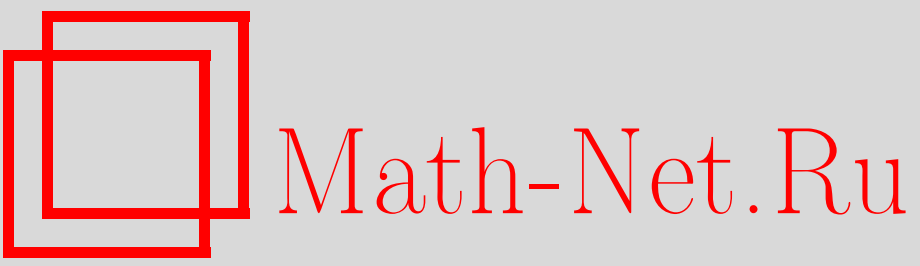

В. Ф. Павлов, В. А. Кирпичёв, А. В. Чирков, О. Ю. Семёнова, Закономерности распределения дополнительных остаточных напряжений в упрочнённых цилиндрических деталях с кольцевыми надрезами полукруглого профиля, Becmн. Сам. гос. техн. ун-та. Сер. Физ.-мат. науки, 2010, выпуск 1(), 121-126

DOI: https://doi.org/10.14498/vsgtu787

Использование Общероссийского математического портала Math-Net.Ru подразумевает, что вы прочитали и согласны с пользовательским соглашением

http://www.mathnet.ru/rus/agreement

Параметры загрузки:

IP : 3.89 .197 .203

26 апреля 2023 г., $14: 38: 30$ 
УДК 539.4.014

\title{
ЗАКОНОМЕРНОСТИ РАСПРЕДЕЛЕНИЯ ДОПОЛНИТЕЛЬНЫХ ОСТАТОЧНЫХ НАПРЯЖЕНИЙ В УПРОЧНЁННЫХ ЦИЛИНДРИЧЕСКИХ ДЕТАЛЯХ С КОЛЬЦЕВЫМИ НАДРЕЗАМИ ПОЛУКРУГЛОГО ПРОФИЛЯ
}

\author{
В. Ф. Павлов, В. А. Кирпичёв, А.В. Чирков, О. Ю. Семёнова
}

Самарский государственный аэрокосмический университет им. ак. С. П. Королёва, 443086, Самара, Московское ш., 34.

E-mail: sopromat@ssau.ru

\begin{abstract}
Изучены закономерности распределения дополнительных остаточных напряжений в упрочнённых полых и сплошных иилиндрических деталях после нанесения кольцевых надрезов полукруглой формы с различными вариантами изменения остаточных усилий гладких деталей.
\end{abstract}

Ключевые слова: дополнителъные остаточные напряжения, поверхностное упрочнение, иилиндрическая деталь, полукругльй кольцевой надрез.

Большое влияние на сопротивление усталости деталей оказывают остаточные напряжения, поэтому в практике современного машиностроения широкое применение находят различные методы поверхностного упрочнения, создающие в поверхностном слое деталей сжимающие остаточные напряжения. Особенно эффективно упрочнение для деталей с концентраторами напряжений, причём этот эффект сохраняется и в случае нанесения концентратора на предварительно упрочнённую деталь [1]. Для установления связи между пределом выносливости упрочнённой и неупрочнённой деталей необходимо знать остаточные напряжения в наименьшем сечении упрочнённой детали с концентратором напряжений [2].

В работе рассматриваются остаточные напряжения в цилиндрической полой детали с единичным надрезом полукруглой формы, часто встречающимся в практике машиностроения. На предварительно упрочнённую методом поверхностного пластического деформирования (ППД) гладкую деталь с наружным диаметром $D$ и внутренним $d$

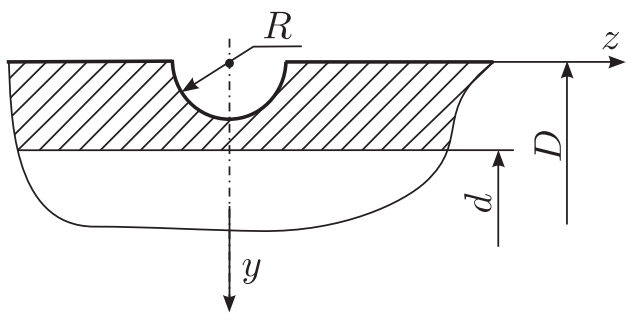
(рис. 1) безнаклёпным способом [1]

Рис. 1. Надрез полукруглого профиля на цинаносится надрез радиуса $R$.

В результате в области детали, прилегающей к надрезу, происходит перераспределение остаточных усилий и возникают дополнительные остаточные напряжения. Известен способ определения этих напряжений для мелких надрезов полукруглого и полуэллиптического профилей с помощью аналитиче-

Валентин Фёдорович Павлов (д.т.н., проф.), зав. кафедрой, каф. сопротивления материалов. Виктор Алексеевич Кирпичёв (к.т.н., доц.), доцент, каф. сопротивления материалов. Алексей Викторович Чирков, аспирант, каф. сопротивления материалов. Олъга Юръевна Семёнова, ст. преподаватель, каф. высшей математики. 
ских зависимостей $[3,4]$. Конечное выражение для дополнительных осевых остаточных напряжений $\sigma_{z д}^{0}$ на дне полукруглого надреза имеет вид [4]

$$
\sigma_{z \text { д }}^{0}=\int_{0}^{1}\left(\frac{4}{\pi \sqrt{1-\xi^{2}}}+0,0925 \sqrt{1-\xi^{2}}\right) \sigma_{z}(\xi) d \xi
$$

где $\sigma_{z}(\xi)$ - осевое остаточное напряжение гладкой детали, $\xi=y / R$ - расстояние от цилиндрической поверхности до текущего слоя, выраженное в долях $R$.

Распределение дополнительных остаточных напряжений в наименьшем сечении цилиндрической детали можно определить по приближённой зависимости

$$
\sigma_{z \text { д }}(\xi)=\frac{\xi^{2}+3}{4 \xi^{4}} \sigma_{z \text { д }}^{0},
$$

которая получена из точного решения [4] путём отбрасывания уточняющих членов. Формула (2) совпадает с решением Г. Нейбера [5] для растянутого образца с надрезом полукруглого профиля.

Формулы (1) и (2) часто используются для определения остаточных напряжений в наименьшем сечении образца с надрезом. Возникает вопрос: насколько достоверными можно считать результаты вычислений по зависимостям (1) и (2) для деталей конечных размеров и надрезов, соизмеримых с толщиной стенки цилиндра? В связи с этим задача теории упругости о перераспределении остаточных усилий гладкой детали решалась численным методом - методом конечных элементов.

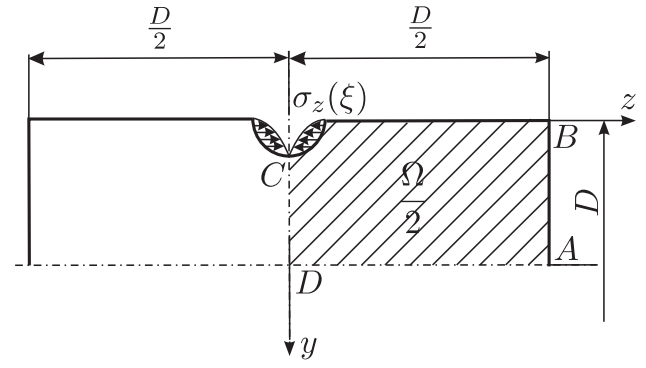

Рис. 2. Расчётная область детали

Границы расчётной области выбирались исходя из принципа СенВенана, то есть расчётная область ограничивалась размером $D / 2$ (рис. 2), где $D$ - наружный диаметр цилиндрической детали. При этом считалось, что за пределами $D / 2$ напряжения в детали для нагружения, показанного на рис. 2, принимают значения, равные нулю. Исходя из условия осевой симметрии нагружения и геометрии, две другие стороны расчётной области ограничивались осью симметрии и поверхностью детали.

Нагружение расчётной области проводилось силами, приложенными на поверхности концентратора вдоль оси $z$, численно равными осевым остаточным напряжениям гладкой детали $\sigma_{z}(\xi)$, но направленными в противоположную сторону. Из условия симметрии рассматривалась только половина расчётной области $\Omega / 2$ с соответствующими геометрическими граничными условиями - граница $C D$ (рис. 2) фиксировалась вдоль оси $z$. Используемая конечно-элементная идеализация позволяла рассматривать цилиндрические детали с различными значениями $D, d$ и $R$ (рис. 1 ). Исследовались сплошные цилиндры с диаметром $D=10 \div 50$ мм, а также полые цилиндры с диаметром отверстия $d=5 \div 12 \mathrm{мм}(D=15 \mathrm{мm}), d=10 \div 19 \mathrm{мm}(D=25 \mathrm{мm})$, $d=30 \div 40 \mathrm{Mм}(D=50 \mathrm{mм})$. 
Рассматривались различные варианты эпюр осевых остаточных напряжений $\sigma_{z}(\xi)$ гладкой детали (рис. 3), охватывающие практически все возможные случаи их распределения после упрочнения деталей известными методами ППД. Варианты 1-5 соответствуют обработке ППД с интенсивными режимами упрочнения, а также деталей из материалов с низкой температу-

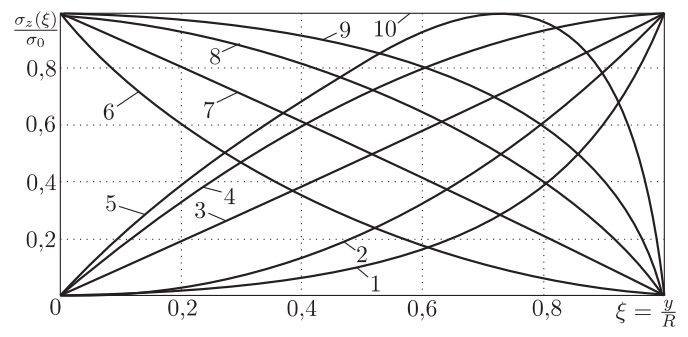

Рис. 3. Варианты распределения остаточных напряжений в поверхностном слое гладкой детали ропроводностью [6], когда наблюдается спад сжимающих остаточных напряжений к поверхности, то есть имеет место случай подповерхностного максимума напряжений. Такие эпюры реализуются, например, при обкатке роликом с большими усилиями накатывания. Упрочнение деталей на более мягких режимах или деталей, изготовленных из материалов с высокой температуропроводностью, приводит к распределению остаточных напряжений по вариантам 6-9, когда максимум напряжений находится на поверхности детали. Вариант 10 соответствует случаю мелкого надреза, когда напряжения можно считать постоянными по толщине поверхностного слоя детали, равной $R$.

В таблице представлены аналитические выражения приведённых на рис. 3 вариантов распределения остаточных напряжений, причём нумерация вариантов таблицы соответствует нумерации эпюр на рис. 3 , а $\sigma_{0}$ - максимальное значение остаточных напряжений.

Результаты вычислений дополнительных остаточных напряжений $\sigma_{z \text { д }}^{0}$ на дне надреза $R=0,3$ мм по формуле (1) и численным методом для сплошных образцов диаметром 10 мм содержатся в таблице. Можно видеть, что наибольшее расхождение при расчёте $\sigma_{z д}^{0}$ двумя способами составляет $12-14 \%$ для вариантов 1 и 2 распределения остаточных напряжений, когда на поверхности детали остаточные напряжения равны нулю, а эпюра имеет наименьшую полноту. Аналогичные результаты получены и для больших диаметров

Варианты распределения остаточных напряжений гладкой детали и дополнительные остаточные напряжения на дне надреза полукруглой формы

\begin{tabular}{c|c|c|c|c}
\hline Вариант & $\sigma_{z}(\xi) / \sigma_{0}$ & $\begin{array}{c}\sigma_{z \text { д }}^{0} / \sigma_{0} \\
\text { по формуле } \\
(1)\end{array}$ & $\begin{array}{c}\sigma_{z \text { д }}^{0} / \sigma_{0} \\
\text { численным } \\
\text { методом }\end{array}$ & Расхождение, \% \\
\hline 1 & $1-\sqrt{1-\xi^{2}}$ & 0,74 & 0,65 & 14 \\
2 & $\xi^{2}$ & 1,02 & 0,91 & 12 \\
3 & $\xi$ & 1,30 & 1,21 & 7 \\
4 & $1-(1-\xi)^{2}$ & 1,59 & 1,48 & 7 \\
5 & $2 \xi \sqrt{1-\xi^{2}}$ & 1,32 & 1,27 & 4 \\
6 & $(1-\xi)^{2}$ & 0,48 & 0,51 & 5 \\
7 & $1-\xi$ & 0,77 & 0,81 & 3 \\
8 & $1-\xi^{2}$ & 1,05 & 1,08 & 0 \\
9 & $\sqrt{1-\xi^{2}}$ & 1,34 & 1,34 & 2,5 \\
10 & 1 & 2,07 & 2,02 & 5 \\
\hline
\end{tabular}


(до 50 мм) цилиндрических деталей и радиусов надрезов (до $R=1,0$ мм), причём с увеличением диаметра $D$ при одном и том же радиусе надреза $R$ расхождение уменьшается. Необходимо отметить, что распределение остаточных напряжений, подобное вариантам 1 и 2 , на практике встречается весьма редко, поэтому можно считать, что расхождение при расчёте $\sigma_{z д}^{0}$ по формуле (1) и численным методом по данным таблицы не превышает $10 \%$.

Для некоторых вариантов рас-

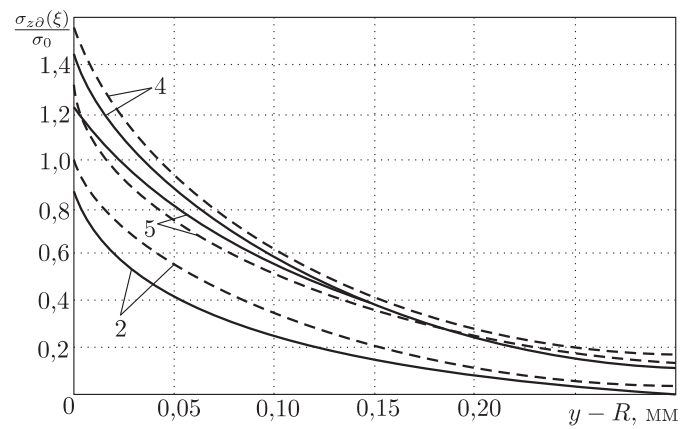

Рис. 4. Распределение дополнительных остаточных напряжений $\sigma_{z \text { д }}(\xi)$ в наименьшем сечении цилиндра $D=10$ мм с надрезом $R=$ $=0,3$ мм, вычисленных: штриховая линия - по формуле (2), сплошная линия - численным методом

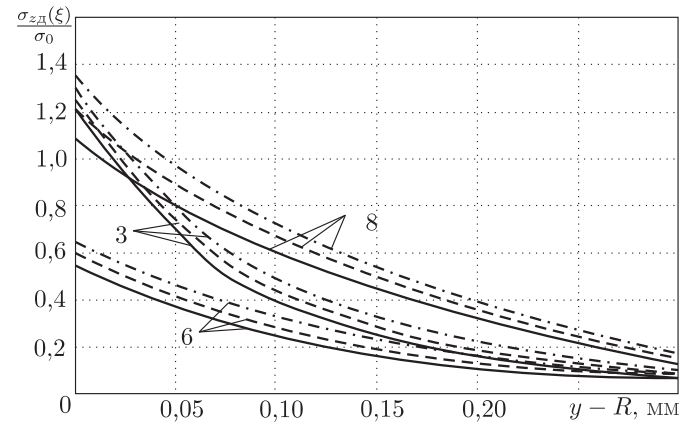

Рис. 5. Распределение дополнительных остаточных напряжений $\sigma_{z \text { д }}(\xi)$ в наименьшем сечении полого цилиндра $D=15$ мм с надрезом $R=0,3$ мм: сплошная линия $-d=5$ мм, пунктирная линия $-d=10$ мм, штрих-пунктирная линия $d=12 \mathrm{MM}$ пределения остаточных напряжений (варианты 2, 4, 5) гладкой детали на рис. 4 представлены результаты расчётов $\sigma_{z \text { д }}(\xi)$ до глубины 0,3 мм по формуле (2) и численным методом для образцов диаметром 10 мм с радиусом надреза $R=0,3$ мм. Из приведённых на рис. 4 данных следует, что наибольшее расхождение наблюдается для варианта 2, причём относительное расхождение увеличивается по мере удаления от поверхности надреза, тогда как абсолютное или остаётся постоянным, или уменьшается. Эта тенденция наблюдается в той или иной мере для всех вариантов распределения остаточных напряжений. В случае наиболее часто встречающихся на практике вариантов эпюр остаточных напряжений, например, для варианта 5, как относительное, так и абсолютное расхождение по толщине поверхностного слоя до 0,3 мм не превышает $17 \%$. Необходимо обратить внимание на то, что при расчёте по формуле (2) дополнительные остаточные напряжения по толщине детали оказываются завышенными.

Исследовались также закономерности распределения дополнительных остаточных напряжений в наименьшем сечении цилиндрической детали с отверстием. На рис. 5 в качестве примера для вариантов 3,6 и 8 представлены графики изменения $\sigma_{z \text { д }}(\xi)$ до 0,3 мм в полых цилиндрах $(D=15$ мм, $R=0,3$ мм) с внутренним диаметром $d=5,10,12$ мм, а на рис. 6 - распределение $\sigma_{z д}(\xi)$ в полых цилиндрах $(D=$ $=25 \mathrm{мм}, R=0,3$ мм) с внутренним диаметром $d=10,14,19$ мм. Оценивая эти результаты, можно сделать вывод, что в исследованных промежутках внутренних диаметров $d$ концентрация остаточных напряжений с уменьшением толщины стенки цилиндра $h=(D-d) / 2$ возрастает и тем существеннее, чем 
тоньше стенка. Так, для варианта 8 (рис. 5) при изменении $h$ от 5 до 1,0 мм $\sigma_{z \text { д }}(\xi)$ возрастают на $42 \%$ как на поверхности, так и по толщине наименьшего сечения детали, вплоть до 0,3 мм.

На рис. 7 для вариантов 2, 5 и 9 приведены результаты расчётов $\sigma_{z д}(\xi)$ для полых цилиндров постоянной толщины стенки $h=$ $=5 \mathrm{mм} \mathrm{c}$ наружным диаметром $D=10,15,25,50$ мм и радиусом надреза $R=0,3$ мм. Видно, что в этом случае и на поверхности концентратора, и по толщине наименьшего сечения детали дополнительные остаточные напряжения практически одинаковы для цилиндров c различными внешними диаметрами. Аналогичные результаты получены для всех других вариантов распределения остаточных напряжений и радиусов надреза $R=$ $=0,5$ мм и $R=1,0$ мм. Таким образом, для исследованных значений $D$ и $R$ на дополнительные остаточные напряжения оказывает влияние только толщина стенки цилиндра.

Было изучено также влияние радиуса надреза $R$ на распределение $\sigma_{z \text { д }}(\xi)$ в наименьшем сечении сплошного цилиндра $D=10$ мм. При этом предполагалось, что остаточные напряжения гладкой детали $\sigma_{z}(\xi)$ меняли свой знак при $y=R$, то есть на дне надреза. Результаты исследования приведены на рис. 8 применительно к варианту 5 распределения $\sigma_{z}(\xi)$. Из приведённых на рис. 8 данных следует, что дополнительные остаточные напряжения $\sigma_{z}(\xi)$ возрастают с увеличением радиуса надреза. Наблюдаемое явление в данном случае объясняется тем, что существенную роль в изменении распределения $\sigma_{z}(\xi)$ играет условие смены знака остаточных напряжений гладкой детали $\sigma_{z}(\xi)$ на дне надреза, в результате чего с увеличением радиуса надреза увеличивается и равнодействующая приложенной нагрузки.

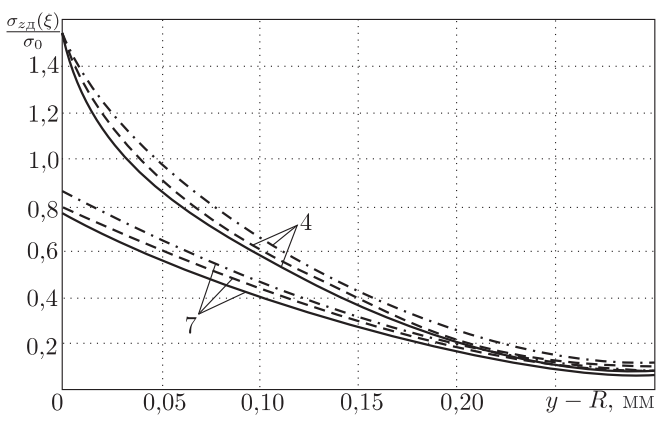

Рис. 6. Распределение дополнительных остаточных напряжений $\sigma_{z \text { д }}(\xi)$ в наименьшем сечении полого цилиндра $D=25$ мм с надрезом $R=0,3$ мм: сплошная линия $-d=10$ мм, штриховая линия $-d=14$ мм, штрих-пунктирная линия $-d=19$ мм

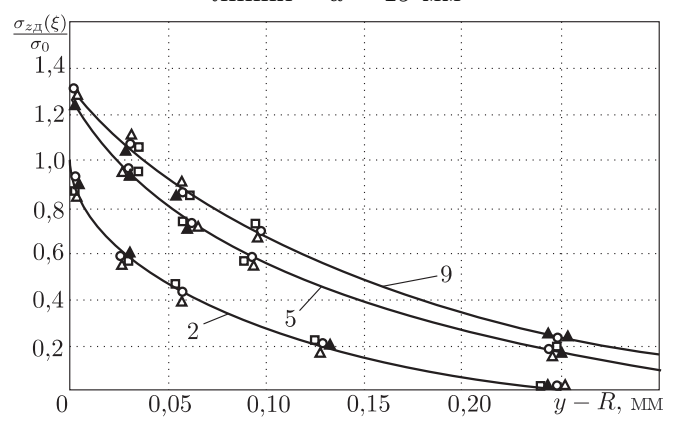

Рис. 7. Распределение дополнительных остаточных напряжений $\sigma_{z \text { д }}(\xi)$ в наименьшем сечении полого цилиндра с надрезом $R=0,3$ мм: $\triangle-D=10 \mathrm{мм}, d=0 ; \circ-D=15 \mathrm{~mm}, d=5 \mathrm{~mm} ;$ $\boldsymbol{\Lambda}-D=25 \mathrm{mм}, d=15 \mathrm{~mm} ; \square-D=50 \mathrm{mм}, d=$ $=40 \mathrm{Mm}$

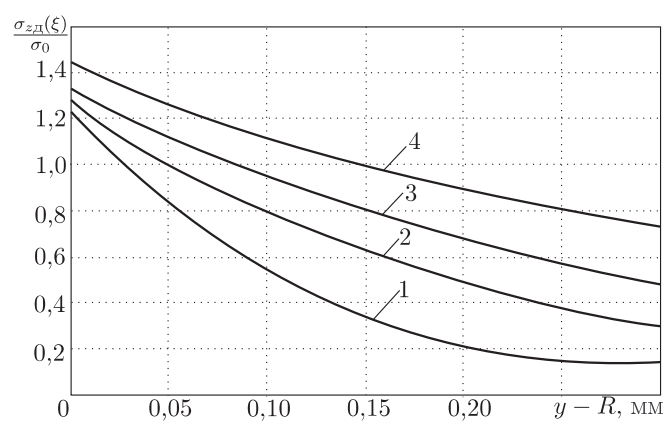

Рис. 8. Распределение дополнительных остаточных напряжений $\sigma_{z \text { д }}(\xi)$ в наименьшем сечении сплошного цилиндра $D=10$ мм: $1-$ $4-\bar{R}=1,0 \mathrm{MM}$ $R=0,3 \mathrm{~mm} ; 2-R=0,5 \mathrm{mM} ; 3-R=0,7 \mathrm{mM} ;$ 


\section{Выводы.}

1. Для наиболее часто встречающихся на практике вариантов распределения остаточных напряжений гладкой детали расхождение результатов расчёта дополнительных остаточных напряжений на дне надреза $\sigma_{z \text { д }}^{0}$ по формуле (2) и численным методом не превышает $10 \%$, а при $y>R$ расхождение повышается с увеличением расстояния от поверхности надре3а.

2. С уменьшением толщины стенки цилиндра с надрезом дополнительные остаточные напряжения в его наименьшем сечении непрерывно возрастают.

3. Дополнительные остаточные напряжения в наименьшем сечении полого цилиндра с надрезом зависят только от толщины стенки и закона распределения остаточных напряжений гладкой детали.

Работа выполнена при поддержке Федерального агентства по образованию (код проекта РНП 2.1.1/3397).

\section{БИБЛИОГРАФИЧЕСКИЙ СПИСОК}

1. Иванов С. И., Павлов В. Ф. Влияние остаточных напряжений и наклёпа на усталостную прочность // Пробл. прочности, 1976. - № 5. - С. 25-27.

2. Павлов В.Ф., Кирпичёв В.А., Иванов В.Б. Остаточные напряжения и сопротивление усталости упрочнённых деталей с концентраторами напряжений. - Самара: СНЦ РАН, 2008. - 64 c.

3. Иванов С. И., Шатунов М. П., Павлов В. Ф. Определение дополнительных остаточных напряжений в надрезах на цилиндрических деталях / В сб.: Bonросы прочности элементов авиачионных конструкиий. Вып. 60. - Куйбышев: КуАИ, 1973. - С. 160-170.

4. Иванов С.И., Шатунов М. П., Павлов В.Ф. Влияние остаточных напряжений на выносливость образцов с надрезом / В сб.: Вопросы прочности элементов авиационных конструкиий. Вып. 1. - Куйбышев: КуАИ, 1974. - С. 88-95.

5. Нейберг Г. Концентрация напряжений. - М.: ОГИЗ, 1947. -214 с.

6. Павлов В.Ф. Влияние теплофизических характеристик материала на распределение остаточных напряжений у поверхности детали// Вестн. машиностроения, 1986. № 5 . - C. $23-24$.

MSC: 74A10, 74C05

Поступила в редакцию 27/II/2010; в окончательном варианте - 25/III/2010.

\section{REGULARITY OF AUXILIARY RESIDUAL STRESSES \\ DISTRIBUTION INTO THE SURFACE TREATED CYLINDRICAL DETAILS WITH ANNULAR SEMICIRCULAR NOTCHES}

\section{V.F. Pavlov, V.A. Kirpichyov, A. V. Chirkov, O. Yu. Semyonova}

S. P. Korolyov Samara State Aerospace University, 34, Moskovskoe sh., Samara, 443086.

E-mail: sopromat@ssau.ru

Regularity of distribution of auxiliary residual stresses in the surface treated hollow and solid cylindrical details after putting of annular semicircular notches with different residual forces variation into even details was researched.

Key words: auxiliary residual stresses, surface treating, cylindrical detail, semicircular.

Original article submitted $27 / \mathrm{II} / 2010$; revision submitted $25 / \mathrm{III} / 2010$.

Valentin F. Pavlov (Dr. Sci. (Tech.)), Head of Dept., Dept. of Material Resistance.

Victor A. Kirpichyov (Ph. D. (Techn.)), Associate Professor, Dept. of Material Resistance. Aleksey V. Chirkov, Postgraduate Student, Dept. of Material Resistance. Olga Yu. Semyonova, Lecturer, Dept. of Higher Mathematics. 